J Am Chem Soc. 2018 December 19; 140(50): 17456-17464. doi:10.1021/jacs.8b07607.

\title{
Imaging Insulin Secretion from Mouse Pancreas by MRI Is Improved by Use of a Zinc-Responsive MRI Sensor with Lower Affinity for $\mathrm{Zn}^{2+}$ lons
}

\author{
André F. Martins ${ }^{\dagger, \ddagger, \|}$, Veronica Clavijo Jordan ${ }^{\ddagger, \|}$, Filip Bochner ${ }^{\S}, \|$, Sara Chirayil ${ }^{\ddagger}$, Namini \\ Paranawithana $^{\dagger}$, Shanrong Zhang ${ }^{\ddagger}$, Su-Tang Lo ${ }^{\ddagger}, X^{\prime a o d o n g}$ Wen $^{\ddagger}$, Piyu Zhao ${ }^{\dagger}$, Michal \\ Neeman $\S$, and A. Dean Sherry ${ }^{*},+\ddagger$ \\ † Department of Chemistry and Biochemistry, University of Texas at Dallas, Richardson, Texas \\ 75080, United States \\ ‡ Advanced Imaging Research Center, University of Texas Southwestern Medical Center, Dallas, \\ Texas 75390-8568, United States \\ $\S$ Department of Biological Regulation, The Weizmann Institute of Science, 7610001 Rehovot, \\ Israel
}

\section{Abstract}

\begin{abstract}
It has been demonstrated that divalent zinc ions packaged with insulin in $\beta$-cell granules can be detected by MRI during glucose-stimulated insulin secretion using a gadolinium-based $\mathrm{Zn}^{2+}$ sensitive agent. This study was designed to evaluate whether a simpler agent design having single $\mathrm{Zn}^{2+}$-sensing moieties but with variable $\mathrm{Zn}^{2+}$ binding affinities might also detect insulin secretion from the pancreas. Using an implanted MR-compatible window designed to hold the pancreas in a fixed position for imaging, we now demonstrate that focally intense "hot spots" can be detected in the tail of the pancreas using these agents after administration of glucose to stimulate insulin secretion. Histological staining of the same tissue verified that the hot spots identified by imaging correspond to clusters of islets, perhaps reflecting first-responder islets that are most responsive to a sudden increase in glucose. A comparison of images obtained when using a high-affinity $\mathrm{Zn}^{2+}$ sensor versus a lower-affinity sensor showed that the lower-affinity sensors produced the best image contrast. An equilibrium model that considers all possible complexes formed between $\mathrm{Zn}^{2+}$, the GdL sensor, and HSA predicts that a GdL sensor with lower affinity for $\mathrm{Zn}^{2+}$ generates a lower background signal from endogenous $\mathrm{Zn}^{2+}$ prior to glucose-stimulated insulin secretion (GSIS) and that the weaker binding affinity agent is more responsive to a further increase in $\mathrm{Zn}^{2+}$ concentration near $\beta$-cells after GSIS. These model predictions are consistent with the in vivo imaging observations.
\end{abstract}

\footnotetext{
*Corresponding Author: dean.sherry@utsouthwestern.edu; sherry@utdallas.edu.

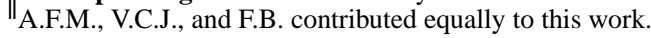

The authors declare no competing financial interest.

ASSOCIATED CONTENT

Supporting Information

The Supporting Information is available free of charge on the ACS Publications website at DOI: 10.1021/jacs.8b07607. Synthetic details for preparing the Gd(III) complexes, the experimental details of the relaxometric experiments, fitted ${ }^{17} \mathrm{O}$ NMR experiments, titration computational details, and mathematical models (PDF)
} 


\section{Graphical Abstract}

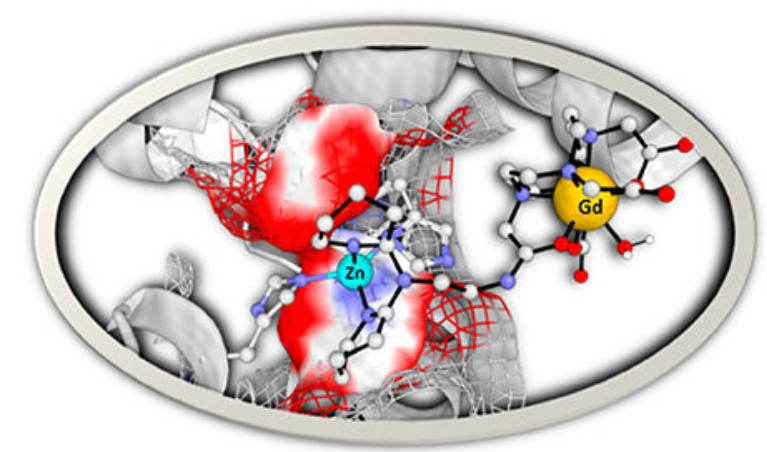

\section{INTRODUCTION}

Contrast agents (CAs) are widely used in magnetic resonance imaging (MRI) diagnostic studies for measuring a variety of dynamic and functional processes. Gadolinium-based $\left(\mathrm{Gd}^{3+}\right)$ CAs are most common due to their chemical stability, rapid clearance, and flexible design. ${ }^{1}$ Most $\mathrm{Gd}^{3+}$ contrast agents in current use are nonspecific extracellular agents, except for a few blood-pool agents that remain in the vascular space longer than others because of weak interactions with serum albumin. ${ }^{2}$ A number of different approaches have been taken over the past two decades to develop agents that respond to physiology, or that can be targeted to specific biological structures. ${ }^{3}$ Recent examples of biologically responsive agents are those that "turn on" in the presence of high concentrations of divalent zinc $\left(\mathrm{Zn}^{2+}\right)$. ${ }^{4,5} \mathrm{Zn}^{2+}$ ions play an essential role in many cellular processes, including structural, catalytic, and signal transduction. Imbalances in tissue $\mathrm{Zn}^{2+}$ content have been associated with Alzheimer's disease, prostate cancer, infertility, and diabetes. ${ }^{6-8}$ The fact that the distribution of $\mathrm{Zn}^{2+}$ is tightly regulated in mammalian tissues highlights the importance of this ion in many biological processes. ${ }^{9-11} \mathrm{Zn}^{2+}$ is present in blood at relatively low concentrations (high nanomolar to low micromolar and mostly bound to protein), but in some tissues, including the pancreas, prostate, cervix, and brain, $\mathrm{Zn}^{2+}$ is present in much higher concentrations (millimolar). ${ }^{12,5,13}$ For example, it is well-known that pancreatic $\beta$ cells store insulin in granules with weakly bound $\mathrm{Zn}^{2+}$ and upon release of insulin, the local concentration of $\mathrm{Zn}^{2+}$ around $\beta$-cells transiently rises to $\sim 450 \mu \mathrm{M}$. ${ }^{14,12}$ The released $\mathrm{Zn}^{2+}$ is also thought to act as a second messenger to communicate with other cell types in pancreatic islets. ${ }^{12,15}$ Several types of $\mathrm{Zn}^{2+}$ sensors have been reported, ${ }^{5,6-18}$ but only a few have been demonstrated to work in vivo. ${ }^{19,20}$ None have been applied clinically. Optical agents detect $\mathrm{Zn}^{2+}$ with high sensitivity, but optical imaging is largely confined to cell-imaging studies due to the low penetration of light through tissues in vivo. ${ }^{4,21,15,16}$ Although less sensitive than optical methods, MRI offers the advantage of unlimited tissue penetration, excellent spatial resolution, and the opportunity to provide functional information. However, the first zinc-responsive CAs for MRI have been largely proof- of-concept reports. ${ }^{22,23,17,24}$

GdDOTA-diBPEN (BPEN = bis-pyridyl-ethylamine), our first-generation $\mathrm{Zn}^{2+}$-responsive MR contrast agent, ${ }^{25}$ has two appended BPEN ligands, each having a high affinity for $\mathrm{Zn}^{2+}$. The resulting ternary complex then and only then binds with human serum albumin (HSA), 
resulting in an increase in $r_{1}$ water proton relaxivity from 5.0 to $17.5 \mathrm{mM}^{-1} \mathrm{~s}^{-1}$ at $0.5 \mathrm{~T}^{5,25}$ Even though the change in $r_{1}$ upon binding with HSA is smaller at $9.4 \mathrm{~T}$, this difference was still sufficient to detect the release of $\mathrm{Zn}^{2+}$ ions from pancreatic $\beta$-cells in vivo by MRI. ${ }^{26}$ The relaxivity of this class of agents was later improved by simple chemical manipulations to increase the rate of water exchange $\left(k_{\mathrm{ex}}\right)$ between the single inner-sphere water molecule on the $\mathrm{Gd}^{3+}$ ion with bulk solvent. ${ }^{20}$ This optimization resulted in a remarkable improvement in $r_{1}$ to $50 \mathrm{mM}^{-1} \mathrm{~s}^{-1}$ measured at $0.5 \mathrm{~T}$. Further in vivo studies in mice using these $r_{1}$-optimized agents showed a nicely enhanced pancreas after an agent was continuously infused into a mouse for $\sim 30 \mathrm{~min}$ followed by a bolus of $20 \% \mathrm{w} / \mathrm{v}$ glucose. ${ }^{20}$ Nonetheless, a quantitative imaging comparison of these new high- $r_{1}$ agents with the original GdDOTA-diBPEN using an identical infusion protocol indicated that image contrast enhancement was not significantly improved for the higher- $r_{1}$ agents $(p<0.125) .{ }^{20}$ This observation suggests that the $r_{1}$ relaxivity of the agent is not the primary determinant in limiting the detection sensitivity of $\mathrm{Zn}^{2+}$ secreted from pancreatic islets in vivo. Given that islets represent a small volume fraction of the pancreas, ${ }^{27}$ one would anticipate that image enhancement due to insulin and $\mathrm{Zn}^{2+}$ release from $\beta$-cells might only be detected in those regions of the pancreas with the highest number of islets. However, not all sections of the mouse pancreas are clearly identifiable in vivo by MRI and this, along with severe respiratory motion, makes high-resolution imaging of the mouse pancreas challenging. Window chamber preparations have been commonly employed in the past to conduct longitudinal optical imaging of internal organs in mice, including brain, ${ }^{28}$ spinal chord, ${ }^{29}$ bone tissue, ${ }^{30}$ and lymph nodes, ${ }^{31}$ as well as liver, spleen, intestine, kidney, and pancreas. ${ }^{32}$ In this study, we used a novel nonmagnetic window adapted from an ovarian window originally designed for optical imaging ${ }^{33}$ sutured into the abdomen of mice to help restrict movement of the pancreas tail. The convenient design of the window also aided comparison of MR imaging slices with tissue slices collected for histology. Using this device, we demonstrated that $\mathrm{Zn}^{2+}$ ion secretion from $\beta$-cells can be detected as high intensity, focal "hot spots" in the pancreas tail only after glucose is given to stimulate insulin and $\mathrm{Zn}^{2+}$ secretion. A comparison of two new $\mathrm{Zn}^{2+}$ sensors having a single binding moiety, one with high affinity for $\mathrm{Zn}^{2+}$ and one with lower affinity for $\mathrm{Zn}^{2+}$, revealed that while both agents detect local "hot spots" in the pancreas tail, the agent with reduced affinity for $\mathrm{Zn}^{2+}$ provided the best focal hot spot intensity versus background after stimulation of insulin secretion.

\section{RESULTS AND DISCUSSION}

The structures of the two agents reported here, GdL1 and GdL2, are shown in Figure 1. The synthetic details of their preparation are given in the Supporting Information (SI). Briefly, the strategy used to alter the binding affinity for zinc was to use a modified BPEN structure, [2-(pyridin-2-yl)ethyl](pyridin-2-ylmethyl)amine or PEPMA, a ligand known to have a lower affinity for $\mathrm{Zn}^{2+}$. The $r_{1}$ relaxivities of GdL1 and GdL2, their binary complexes with $\mathrm{Zn}^{2+}$, and their ternary complexes with both $\mathrm{Zn}^{2+}$ and HSA are listed in Table 1. These data show that both GdL1 and GdL2 have relaxation characteristics similar to those displayed by our first-generation agents, i.e., $r_{1}$ increases only in the presence of both $\mathrm{Zn}^{2+}$ and HSA. Interestingly, the magnitude of $r_{1}$ for GdL1 in the presence of both $\mathrm{Zn}^{2+}$ and HSA was 
similar to that observed for GdDOTA-diBPEN, ${ }^{25,5}$ while the $r_{1}$ of GdL2 is $\sim 40 \%$ lower at $0.5 \mathrm{~T}$. A titration of each complex with $\mathrm{Zn}^{2+}$ showed that $r_{1}$ increased only slightly upon formation of a 1:1 complex with $\mathrm{Zn}^{2+}$ in buffer [Table 1 and Figure S1 (SI)], while in the presence of $600 \mu M \mathrm{HSA}$, the $r_{1}$ of GdL1 and GdL2 increased by 3.7- and 2.4-fold, respectively. These data show that the sensors reported here having a single $\mathrm{Zn}^{2+}$ binding moiety retain their ability to bind with HSA in the presence of $\mathrm{Zn}^{2+}$ ions, similar to the previously reported GdDOTA-diBPEN derivatives. ${ }^{20,25}$ Kinetic stability studies also show that these agents are chemically inert toward the release of free $\mathrm{Gd}^{3+}$ ions under extreme conditions (Figure S5, SI).

Human serum albumin, the most abundant protein in plasma, is known to have a relatively high binding affinity for $\mathrm{Zn}^{2+}$ ions $(29.5 \mathrm{nM}) .{ }^{34}$ Molecules containing the BPEN moiety also bind with $\mathrm{Zn}^{2+}$ ions in the nanomolar range $\left(K_{\mathrm{D}(\text { GdDOTA-diBPEN })}=33.6 \mathrm{nM}\right) .{ }^{35,25} \mathrm{In}$ a complex mixture containing a Gd-based zinc sensor (HSA) and $\mathrm{Zn}^{2+}$ ions, several species are present in solution. At low-to-medium magnetic fields, $r_{1}$ is largely governed by the rotational correlation time $\left(\tau_{\mathrm{R}}\right)$. So $r_{1}$, in this case, is dominated by the amount of GdL $x$ - $\mathrm{Zn}$ HSA present in this mixture. Previously reported sensors having a single $\mathrm{Zn}^{2+}$ binding moiety display only modest increases in $r_{1}$ when mixed with albumin from various mammalian sources. ${ }^{17,23,36}$ Given that the rate of water exchange $\left(k_{\mathrm{ex}}\right)$ in these complexes can limit the magnitude of any $r_{1}$ increase when GdL complexes bind to HSA, further ${ }^{17} \mathrm{O}$ NMR relaxation studies were performed to evaluate the water exchange rates. Simultaneous fitting of ${ }^{17} \mathrm{O}$-reduced $T_{1}, T_{2}$, and chemical shift $\left(\Delta \omega_{\mathrm{r}}\right.$ ) data were used to estimate $q$ (number of water exchange sites), $k_{\mathrm{ex}}$, and the hyperfine coupling constant of each complex. The fitted results are summarized in Tables 1 and S1 (SI). The water exchange rates in GdL1 and GdL2 were found to be similar to the value reported for GdDOTA-monoamide derivatives $\left(k_{\mathrm{ex}} \sim 3 \times 10^{6} \mathrm{~s}^{-1}\right)$ despite the fact that these new agents are monoamide derivatives. ${ }^{37-40}$ The $r_{1}$ values of these agents when bound to HSA are somewhat smaller than that observed with GdDOTA-diBPEN (especially true for GdL2), even though the binding affinities with HSA are comparable. This could reflect differences in the solvent structure around the GdL2 binding site that influences the rate of water exchange. To gain more insights into this question, we performed additional ${ }^{17} \mathrm{O}$ NMR experiments using DyL1 and DyL2 in the presence of $0.6 \mathrm{mM}$ HSA and $\mathrm{Zn}^{2+}$ (the $\mathrm{Dy}^{3+}$ complex was used to improve the quality of the ${ }^{17} \mathrm{O}$ data fit at lower agent concentrations; see Figure S3 and Table S2, SI). An analysis of these data shows that the rate of water exchange is about 2-3-fold faster when the complex is bound to HSA compared to that measured for the complex in aqueous solution alone. Although an increase in $k_{\mathrm{ex}}$ to $\sim 9 \times 10^{6} \mathrm{~s}^{-1}$ when GdL1-2 is bound to HSA should be favorable in terms of $r_{1}$, this exchange rate is still about 5 -fold slower than that considered optimal for a maximal increase in $r_{1}$ at common clinical imaging fields $(3 \mathrm{~T}) .{ }^{41,42,1}$

Given that the rodent pancreas is not a solid organ, but a thin tissue interlaced around the intestines, stomach, and spleen, this feature makes it challenging to image the same section of pancreas longitudinally by MRI. This problem is further exacerbated by respiratory motion. For these reasons, we adopted a surgical procedure to implant an imaging window in the mouse abdomen ${ }^{33,43}$ with the goal of holding the tail of the pancreas in a relatively fixed position. As shown in Figure 2A, a custom-made MR-compatible imaging window was sutured in place and the pancreas tail was carefully retracted and held in position with 
two supporting petals directly beneath the window. The spleen was also positioned near the window and used as an anatomical reference. 3D $T_{1}$-weighted MR images (scan time: 3 $\mathrm{min}, 35 \mathrm{~s})$ were collected serially after injection of either GdL1 or GdL2 $(0.07 \mathrm{mmol} / \mathrm{kg})$ plus saline or glucose intravenously. In saline controls, the change in signal intensity relative to preinjection scans across the entire pancreas averaged about 35\% after injection of GdL2 versus about $60 \%$ after injection of an equivalent amount of GdL1 (Figure 2C, top panel). This clearly shows that more GdL $x$-Zn-HSA is formed between endogenous $\mathrm{Zn}^{2+}$ ions and the high-affinity zinc sensor than with the low-affinity zinc sensor. Assuming that the agents themselves do not initiate release of additional $\mathrm{Zn}^{2+}$ ions from endogenous stores, these results indicate that GdL1 produces about a 1.7-fold greater background signal compared to GdL2. The integrated area under the curve over the entire $28 \mathrm{~min}$ for GdL1 versus GdL2 in the saline-only images (no glucose) was significantly lower for GdL2 ( $p$-value $<0.05$ ) (Figure 2C, bottom panel). After coinjection of agent plus glucose, both agents resulted in even greater image enhancement over the background (Figure 2C, middle panel), but interestingly, contrast enhancement was not uniform across the pancreas with either agent. Instead, high intensity focal "hot spots" were observed with both agents but were most evident when using GdL2. These data suggest that the higher background signal produced by GdL1 binding to endogenous $\mathrm{Zn}^{2+}$ partially masks the intense "hot spots" that appear only after stimulation of insulin secretion by glucose. Given the further assumption that the same amount of $\mathrm{Zn}^{2+}$ (and insulin) is released from islets in both experiments and the fact that the $r_{1}$ differences between the two agents are small, these image differences must largely reflect the greater background signal produced by GdL1. Interestingly, the average signal enhancement across the entire pancreas tail is similar for both agents at 3-7 min postinjection of agent and glucose (Figure $2 \mathrm{C}$, middle panel) but then appears to gradually decline starting at $\sim 7$ min for GdL2 but remains relatively high for GdL1 over the entire 28 min experiment, although these apparent differences did not prove to be statistically different for this limited data set. Additional mouse imaging studies and other biological experiments will be required to evaluate any differences in $\beta$-cell biology initiated by these $\mathrm{Zn}^{2+}$-binding agents. Although one can easily discriminate between functional islets and nonsecreting stroma in these images by visual inspection, additional unbiased "hot spot" detection could likely be accomplished by use of machine-learning techniques to further identify differences between GdL2 and GdL1.

In an attempt to confirm that the higher intensity focal "hot spots" detected in the pancreas tail correspond to $\mathrm{Zn}^{2+}$ secretion from $\beta$-cells in islets, we excised the pancreas tail and fixed the tissue with $10 \%$ formalin. This allowed coregistration of the histological sections and MRI slices to the best of our ability. A tissue slice stained with hematoxylin and eosin (H\&E) (Figure 3A) shows that islets are located near highly vascularized regions of the pancreas. The eosinstained islets (labeled as a red overlay) were then superimposed onto the MRI slice that most closely coregistered to the histology slice collected from the same animal (Figure 3B). As shown, the position of the islets as identified by staining only partially overlap the MRI detected hot spots near highly vascularized regions of the pancreas tail. This qualitative observation points out the difficulty of matching the MRI result which reflects a diffusional volume of zinc release (perhaps a reflection of islet function) with the 
exact location and size of individual islets as identified by histology (a reflection of islet mass).

It has been reported that some islets act as "first responders" $" 4,44$ by depleting their entire insulin content in response to an increase in plasma glucose while others release insulin more gradually over longer periods of time. The kinetic curves shown in Figure 2 suggest that the focal hot spots detected in the pancreas within 3-4 min after injection of glucose do reflect early release of insulin from islets, but these data do not allow us to conclude that the hot spots observed here by MRI correspond to "first-responder" islet activity alone or some combination of first responders plus more gradual responders. First-responder islets are by definition ${ }^{37,38}$ those islets that deplete their entire insulin content (and presumably $\mathrm{Zn}^{2+}$ content), but unlike in the optical study of Zhu et al. where C-peptide was labeled with a fluorescent dye, the MRI method reported here detects functional release of $\mathrm{Zn}^{2+}$ but not necessarily $\mathrm{Zn}^{2+}$ content. If one compares the dynamics of $\mathrm{Zn}^{2+}$ release on the basis of averaged intensity of all hot spots (Figure 2C, middle panel) versus intensity from individual hot spots (Figure S11, SI), one finds that individual hot spots appear to show differential $\mathrm{Zn}^{2+}$ secretion (as reflected by MR intensities) and differential rates of $\mathrm{Zn}^{2+}$ secretion. Those islets that respond acutely show a rapid increase in MR signal ( 200\% signal change) while others show a more moderate and slow increase in the signal intensity $(\sim 100 \%)$. While these differences are detectable when using either GdL1 or GdL2, the tissue surrounding secreting islets remains intense over the entire secretory period imaged (36 min) when using GdL1, so any distinction between rapidly responding islets versus gradually responding slets is lost, as any nonspecific background signal masks the true signal from nascent "hotspots". This further validates our hypothesis that the gain in MR signal intensity is significantly higher across the entire pancreas after injection of the high-affinity zinc sensor, GdL1, while the low-affinity zinc sensor, GdL2, more readily discriminates between background versus focal hot spots. Thus, one can conclude that GdL2 provides a better distinction of islets that respond most quickly to an increase in glucose.

In support of the in vivo observations, we performed additional experiments to better understand the biophysical mechanisms behind these observations. HSA is an excellent carrier of drugs, fatty acids, endogenous substances, and metal ions. The protein has three homologous domains (I, II, and III) separated by two helical subdomains, A and B. Prior studies have shown that HSA binds long chain fatty acids in at least seven identified sites (FA1-7) and has two additional drug binding sites, 1 and 2. In addition, three distinct $\mathrm{Zn}^{2+}$ binding sites have also been identified (Figure 4A). The main site for zinc involves Asn-99 and His-67 residues of domain IB and His-247 and Asp-249 of domain IIA. ${ }^{34}$ The latter has been commonly referred to as metal ion binding site A (MBS/site A), but there are also two other zinc binding sites that have been identified by ${ }^{111 / 113} \mathrm{Cd}$ (II) NMR spectroscopy, sites B and $\mathrm{C}$. Site $\mathrm{A}$ is known to bind $\mathrm{Zn}^{2+}$ ions with high affinity, while sites $\mathrm{B}$ and $\mathrm{C}$ have somewhat weaker affinities for $\mathrm{Zn}^{2+} .45,46$ Although these $\mathrm{Zn}^{2+}$ binding sites have been characterized, less is known about structural arrangements that may occur around the metal ion in these sites after addition of a competitive $\mathrm{Zn}^{2+}$ ion binding agent, such as GdL1 or GdL2, to the mixture. To gain further insights into the competition between HSA and these zinc sensors, we performed additional ${ }^{113} \mathrm{Cd}$ NMR experiments using diamagnetic $\operatorname{LaL} x$ as a surrogate for GdL $x$. The bottom ${ }^{113} \mathrm{Cd}$ NMR spectrum in Figure 4B shows that divalent 
$\mathrm{Cd}^{2+}$ binds to the three distinct $\mathrm{Zn}^{2+}$ binding sites. Resonance $\mathrm{A}$ was previously assigned to the high-affinity $\mathrm{Zn}^{2+}$ site referred to as site A. ${ }^{34,46,47}$ After addition of 2 equiv of LaL1 (a diamagnetic analog of GdL1) and $\mathrm{Zn}^{2+}$, the ${ }^{113} \mathrm{Cd}$ resonances of sites $\mathrm{A}$ and $\mathrm{C}$ disappear, showing that $\mathrm{Zn}^{2+}$ displaces $\mathrm{Cd}^{2+}$ from those two sites. This verifies that these two sites have the highest affinity for $\mathrm{Zn}^{2+}$. Conversely, when 1 equiv of $\mathrm{LaL} 1$ (no $\mathrm{Zn}^{2+}$ ) was added, only $\mathrm{Cd}^{2+}$ in site $\mathrm{A}$ was affected. In this case, the resonances characteristic of sites $\mathrm{A}$ and $\mathrm{C}$ broaden, and a new resonance appears downfield of site A near $145 \mathrm{ppm}$. The line width of this new resonance indicates that it also reflects a HSA-bound species, likely $\mathrm{Cd}^{2+}$ in site A with a slightly altered coordination sphere. It has been reported that $\mathrm{Zn}^{2+}$ binding to site $\mathrm{A}$ is altered when fatty acids or other drugs bind at drug site $2 .{ }^{34}$ This was verified here by both ${ }^{113} \mathrm{Cd}$ NMR (Figure S9, SI) and fluorescence displacement experiments using dansylglycine (Figure S6, SI). We conclude from these combined observations that GdL1 and GdL2 bind to $\mathrm{Zn}^{2+}$ ions in site $\mathrm{A}$, and in doing so, the $\mathrm{Zn}^{2+}$ coordination environment is altered such that it becomes more susceptible to displacement by drugs binding in drug site 2. It is most likely that the two pyridine donors in $\operatorname{GdL} x$ bind to $\mathrm{Zn}^{2+}$ in site A by displacement of Asp-249 and Asn-99 from the coordination sphere of $\mathrm{Zn}^{2+}$ (Scheme S3, SI). Molecular modeling (Figure $4 \mathrm{C}$ ) indicates that the resulting ternary structure is energetically quite stable.

To add further support for this binding model, we constructed a mathematical model in MatLab that includes all possible $\operatorname{GdL} x, \mathrm{HSA}$, and $\mathrm{Zn}^{2+}$ binding species, the experimentally determined binding constants, and the measured $r_{1}$ values at 9.4 T reported in Table 1 for each Gd-containing species (Scheme S3, SI). Our goal was to test whether a simple binding model such as this could predict our MRI observation that the lower-affinity $\mathrm{Zn}^{2+}$ binding agent, GdL2, more effectively discriminates islets undergoing GSIS, even though the model may not be quantitative. We assumed the following: (1) a bolus injection of $0.07 \mathrm{mmol} / \mathrm{kg}$ agent yields an extracellular [GdL $x$ ] of $\sim 100 \mu \mathrm{M}$, (2) extracellular [HSA] is $\sim 600 \mu \mathrm{M}$, (3) the concentration of $\mathrm{Zn}^{2+}$ in the extracellular space around $\beta$-cells increases from $\sim 50 \mu \mathrm{M}$ to $\sim 500 \mu \mathrm{M}$ after release of insulin, and (4) the coordination sphere around $\mathrm{Zn}^{2+}$ in metal ion binding site A differs when GdL $x$ is bound at this same site such that the overall binding affinity is reduced. Using this model, one can generate speciation plots such as those shown in Figure S7 (SI). These plots show that when GdL2 is presented to tissues at basal $\mathrm{Zn}^{2+}$ levels $(50 \mu \mathrm{M})$, considerably more Zn-HSA is formed compared to GdL2-Zn-HSA. Conversely, with GdL1, about equal amounts of Zn-HSA and GdL1-Zn-HSA are formed at basal levels, and this results in a substantially higher background MRI signal, as observed experimentally. However, as additional $\mathrm{Zn}^{2+}$ is released from $\beta$-cells, the amount of GdL1$\mathrm{Zn}-\mathrm{HSA}$ that can form reaches a maximum when $\mathrm{Zn}^{2+}$ levels rise to $\sim 100 \mu \mathrm{M}$. This threshold is likely reached very early after an increase in blood glucose saturating the overall signal detected (unknown region represented in Figure 4D). Conversely, using the loweraffinity agent, the amount of GdL2-Zn-HSA formed does not reach its maximum until $\mathrm{Zn}^{2+}$ levels rise to nearly $\sim 450 \mu \mathrm{M}$. Indeed, the binding model predicts that GdL2 has a much higher dynamic range as tissue $\mathrm{Zn}^{2+}$ rises. This is illustrated in Figure 4D. Even if our estimates of total $\mathrm{Zn}^{2+}$ and agent concentration are off by $\pm 10-20 \%$, the equilibrium model presented here correctly predicts the experimental results observed by MRI. 


\section{CONCLUSIONS}

Previous studies from our lab demonstrated that GSIS from the mouse pancreas could be detected by MRI using a Gd-based $\mathrm{Zn}^{2+}$-responsive contrast agent. ${ }^{26,20}$ In those reports, the $\mathrm{Zn}^{2+}$ sensors used were GdDO2A-bis-amide derivatives having two amide side chains with known high-affinity (nM) $\mathrm{Zn}^{2+}$-chelating moieties (BPEN). The unique feature of all $\mathrm{Zn}^{2+}$ responsive agents is the formation of a GdL- $\mathrm{Zn}^{2+}$-HSA ternary complex that displays slower molecular tumbling when GdL- $\mathrm{Zn}^{2+}$ binds to the protein. This results in an increase in water proton relaxivity $\left(r_{1}\right)$ and image enhancement in $T_{1}$-weighted images in those tissues where the local $\mathrm{Zn}^{2+}$ concentration is abnormally high. Given that substantial amounts of $\mathrm{Zn}^{2+}$ are released with insulin during GSIS, we anticipated that we might be able to detect insulin secretion from pancreatic $\beta$-cells using this technology. Indeed, 3D images of the mouse pancreas after injection of $0.07 \mathrm{mmol} / \mathrm{kg}$ of GdDO2A-diBPEN plus glucose showed relatively uniform enhancement of the pancreas. This effect (1) disappeared in animals pretreated with STZ to destroy $\beta$-cells and (2) was more pronounced in animals fed a highfat diet over 12 weeks, consistent with expansion of functional $\beta$-cells in the pancreas. ${ }^{26}$ Both observations supported our hypothesis that insulin secretion from $\beta$-cells can be detected by use of a $\mathrm{Zn}^{2+}$-responsive MRI contrast agent.

The initial goal of the current study was to compare two new GdDO3A-monoamide derivatives having a single $\mathrm{Zn}^{2+}$ chelating group on one sidearm, one with a high-affinity $(\mathrm{nM}) \mathrm{Zn}^{2+}$ binding moiety and another with a lower affinity $(\mu \mathrm{M}) \mathrm{Zn}^{2+}$ binding moiety. The rationale for switching to these one-arm $\mathrm{Zn}^{2+}$ sensors was 2-fold: (1) ease of synthesis and scale-up for studies in larger animal models and (2) higher thermodynamic stability of GdDO3A-monoamides versus GdDO2A-bis-amides. Our results show that both gadolinium derivatives do indeed respond to release of $\mathrm{Zn}^{2+}$ ions from $\beta$-cells. Equally important, we have shown that by using an abdominal window to hold the tail of the rodent pancreas into a relatively fixed position, one can detect focal intensity "hot spots" in the pancreas within minutes after initiation of GSIS. This would not be possible to observe using standard abdominal MRI protocols in mice. The appearance and timing of these image-enhanced regions likely reflect the readily releasable pool of insulin granules that are predocked closest to the $\beta$-cell membrane. ${ }^{48}$

A comparison of the two new agents used in this study showed that the focal hot spots were more clearly demarcated when using the lower-affinity $\mathrm{Zn}^{2+}$ agent, even though the $r_{1}$ of the lower $\mathrm{Zn}^{2+}$ affinity agent when bound to HSA was about $40 \%$ lower compared to the higher $\mathrm{Zn}^{2+}$ affinity agent. This is thought to be due to the lower background signal of tissues having less freely available endogenous $\mathrm{Zn}^{2+}$ and GdL2 being in its "low-relaxivity" form. A similar conclusion was reached previously in a study that compared low- $\Gamma_{1}$ versus high- $r_{1}$ $\mathrm{Zn}^{2+}$-responsive agents. ${ }^{20}$ In that study, it was shown that an agent having a 5-fold increase in $r_{1}$ relaxivity (as measured in vitro) did not translate to a 5-fold increase in sensitivity in vivo. Nonetheless, the images collected here in the mouse pancreas held in a relatively fixed position showed quite clearly that the image differences observed with GdL1 versus GdL2 reflected a higher background signal generated by GdL1, exactly as predicted by the multiequilibrium binding model involving the Gd-based $\mathrm{Zn}^{2+}$ sensors, free $\mathrm{Zn}^{2+}$ ions, and HSA. Similar observations have been reported for other types of metal ion indicators. For 
example, $\mathrm{Li}$ et al. demonstrated that the $\mathrm{Zn}^{2+}$-responsive fluorescence indicator ZIMIR responded optimally to release of intracellular $\mathrm{Zn}^{2+}$ stores when the binding affinity for $\mathrm{Zn}^{2+}$ was reduced. ${ }^{15}$ Similarly, engineered calcitonin-gene-related peptides with lower affinities for $\mathrm{Ca}^{2+}$ were most responsive to changes in $\mathrm{Ca}^{2+}$ levels during an electric stimulation of the mouse brain. ${ }^{49}$

In summary, this study describes an optimized $\mathrm{Zn}^{2+}$-responsive MRI contrast agent for detection of insulin release from pancreatic islets thanks to implementation of a novel MRIcompatible imaging window that stabilized the tail of the pancreas for motion-free imaging. Both the high-affinity $\mathrm{Zn}^{2+}$ agent, GdL1, and the low-affinity $\mathrm{Zn}^{2+}$ agent, GdL2, displayed an increase in $r_{1}$ only in the presence of both $\mathrm{Zn}^{2+}$ and HSA, yet the low-affinity agent gave the most impressive results in vivo. This was traced to a lower background signal produced by the weaker agent. This experimental finding was supported by a mathematical binding model that correctly predicts that the weaker $\mathrm{Zn}^{2+}$ affinity agent has a larger dynamic range in vivo as $\mathrm{Zn}^{2+}$ levels rise during active GSIS. This improved sensitivity results in clear demarcation of focal hot spots within the mouse pancreas, likely reflecting first-responder islets poised to release insulin quickly after an increase in plasma glucose. The exact relationship between the focal hot spots observed by MRI and the population of firstresponder islets will require additional biological experiments, but if this connection can be established, then this imaging method could significantly advance the discovery of new drugs that enhance or modify $\beta$-cell function in vivo.

\section{Supplementary Material}

Refer to Web version on PubMed Central for supplementary material.

\section{ACKNOWLEDGMENTS}

Financial support from the National Institutes of Health (DK-095416 and EB-015908), the American Diabetes Association (ADA 7-12-IN-42), and the Robert A. Welch Foundation (AT-584) is gratefully acknowledged. We also thank Dr. Hien Q. Nguyen for his support and training, performing the ICP-MS studies at UT Dallas, and Dr. James Ratnakar for his helpful support recording the ${ }^{17} \mathrm{O}$ NMR spectra for the Dy complexes at UT Southwestern Medical Center.

\section{REFERENCES}

(1). Merbach AE; Helm L; Toth E The Chemistry of Contrast Agents in Medical Magnetic Resonance Imaging, 2nd ed.; John Wiley \& Sons: Chichester, UK, 2013.

(2). Goyen M Gadofosveset-Enhanced Magnetic Resonance Angiography. Vasc. Health Risk Manag 2008, 4, 1-9. [PubMed: 18629367]

(3). Pierre VC; Allen MJ; Caravan P Contrast Agents for MRI: 30+ Years and Where Are We Going? JBIC J. Biol. Inorg. Chem 2014, 19, 127-131. [PubMed: 24414380]

(4). Zhang X; Lovejoy KS; Jasanoff A; Lippard SJ Water- Soluble Porphyrins as a Dual-Function Molecular Imaging Platform for MRI and Fluorescence Zinc Sensing. Proc. Natl. Acad. Sci. U. S. A 2007, 104, 10780-10785. [PubMed: 17578918]

(5). De Leon-Rodriguez L; Lubag AJM Jr.; Sherry AD Imaging Free Zinc Levels in Vivo — What Can Be Learned? Inorg. Chim. Acta 2012, 393, 12-23.

(6). Cousins RJ Toward a Molecular Understanding of Zinc Metabolism. Clin. Physiol. Biochem 1986, 4, 20-30. [PubMed: 2420502] 
(7). Myers SA; Nield A; Myers M Zinc Transporters, Mechanisms of Action and Therapeutic Utility: Implications for Type 2 Diabetes Mellitus. J. Nutr. Metab 2012, 2012, 173712. [PubMed: 23304467]

(8). Clavijo Jordan MVC; Lo S-T; Chen S; Preihs C; Chirayil S; Zhang S; Kapur P; Li W-H; De LeonRodriguez LMD; Lubag AJM; Rofsky NM; Sherry AD Zinc-Sensitive MRI Contrast Agent Detects Differential Release of Zn(II) Ions from the Healthy vs. Malignant Mouse Prostate. Proc. Natl. Acad. Sci. U. S. A 2016, 113, E5464. [PubMed: 27562169]

(9). Maret W Redox Biochemistry of Mammalian Metallothioneins. JBIC, J. Biol. Inorg. Chem 2011, 16 (7), 1079-1086. [PubMed: 21647775]

(10). Maret W Metallothionein Redox Biology in the Cytoprotective and Cytotoxic Functions of Zinc. Exp. Gerontol 2008, 43, 363-369. [PubMed: 18171607]

(11). Hao Q; Maret W Imbalance between Pro-Oxidant and pro-Antioxidant Functions of Zinc in Disease. J. Alzheimer's Dis 2005, 8, 161-170. [PubMed: 16308485]

(12). Kim BJ; Kim YH; Kim S; Kim JW; Koh JY; Oh SH; Lee MK; Kim KW; Lee MS Zinc as a Paracrine Effector in Pancreatic Islet Cell Death. Diabetes 2000, 49, 367-372. [PubMed: 10868957]

(13). Li YV Zinc and Insulin in Pancreatic Beta-Cells. Endocrine 2014, 45, 178-189. [PubMed: 23979673]

(14). Folin M; Contiero E; Vaselli GM Zinc Content of Normal Human Serum and Its Correlation with Some Hematic Parameters. BioMetals 1994, 7, 75-79. [PubMed: 8118176]

(15). Li D; Chen S; Bellomo EA; Tarasov AI; Kaut C; Rutter GA; Li W Imaging Dynamic Insulin Release Using a Fluorescent Zinc Indicator for Monitoring Induced Exocytotic Release (ZIMIR). Proc. Natl. Acad. Sci. U. S. A 2011, 108, 21063-21068. [PubMed: 22160693]

(16). Stasiuk GJ; Minuzzi F; Sae-Heng M; Rivas C; Juretschke H-P; Piemonti L; Allegrini PR; Laurent D; Duckworth AR; Beeby A; Rutter GA; Long NJ Dual-Modal Magnetic Resonance/Fluorescent Zinc Probes for Pancreatic $\beta$-Cell Mass Imaging. Chem. - Eur. J 2015, 21, 5023-5033. [PubMed: 25736590]

(17). Mishra A; Logothetis NK; Parker D Critical In Vitro Evaluation of Responsive MRI Contrast Agents for Calcium and Zinc. Chem. - Eur. J 2011, 17, 1529-1537. [PubMed: 21268155]

(18). Bonnet CS; Caillé F; Pallier A; Morfin J-F; Petoud S; Suzenet F; Tóth É Mechanistic Studies of Gd3+-Based MRI Contrast Agents for Zn2+ Detection: Towards Rational Design. Chem. - Eur. J 2014, 20, 10959-10969. [PubMed: 25116889]

(19). Lo S-T; Martins AF; Jordan VC; Sherry AD Zinc as an Imaging Biomarker of Prostate Cancer. Isr. J. Chem 2017, 57, 854-861. [PubMed: 30319140]

(20). Yu J; Martins AF; Preihs C; Clavijo Jordan V; Chirayil S; Zhao P; Wu Y; Nasr K; Kiefer GE; Sherry AD Amplifying the Sensitivity of Zinc(II) Responsive MRI Contrast Agents by Altering Water Exchange Rates. J. Am. Chem. Soc 2015, 137, 14173-14179. [PubMed: 26462412]

(21). Xu Z; Yoon J; Spring DR Fluorescent Chemosensors for Zn2+. Chem. Soc. Rev 2010, 39, 1996 2006. [PubMed: 20428518]

(22). Hanaoka K; Kikuchi K; Urano Y; Nagano T Selective Sensing of Zinc Ions with a Novel Magnetic Resonance Imaging Contrast Agent. J. Chem. Soc. Perkin Trans 2 2001, 1840-1843.

(23). Major JL; Parigi G; Luchinat C; Meade TJ The Synthesis and in Vitro Testing of a ZincActivated MRI Contrast Agent. Proc. Natl. Acad. Sci. U. S. A 2007, 104, 13881-13886. [PubMed: 17724345]

(24). Luo J; Li W-S; Xu P; Zhang L-Y; Chen Z-N Zn2+ Responsive Bimodal Magnetic Resonance Imaging and Fluorescent Imaging Probe Based on a Gadolinium(III) Complex. Inorg. Chem 2012, 51, 9508-9516. [PubMed: 22880548]

(25). Esqueda AC; López JA; Andreu-de-Riquer G; Alvarado-Monzón JC; Ratnakar J; Lubag AJM; Sherry AD; De León-Rodríguez LM A New Gadolinium-Based MRI Zinc Sensor. J. Am. Chem. Soc 2009, 131, 11387-11391. [PubMed: 19630391]

(26). Lubag AJM; De Leon-Rodriguez LM; Burgess SC; Sherry AD Noninvasive MRI of?-Cell Function Using a Zn2+- Responsive Contrast Agent. Proc. Natl. Acad. Sci. U. S. A 2011, 108, 18400-18405. [PubMed: 22025712] 
(27). Wang X; Misawa R; Zielinski MC; Cowen P; Jo J; Periwal V; Ricordi C; Khan A; Szust J; Shen J; Millis JM; Witkowski P; Hara M Regional Differences in Islet Distribution in the Human Pancreas - Preferential Beta-Cell Loss in the Head Region in Patients with Type 2 Diabetes. PLoS One 2013, 8, No. e67454. [PubMed: 23826303]

(28). Holtmaat A; Bonhoeffer T; Chow DK; Chuckowree J; De Paola VD; Hofer SB; Hubener M; Keck T; Knott G; Lee WCA; Wilbrecht L; et al. Long-Term, High-Resolution Imaging in the Mouse Neocortex through a Chronic Cranial Window. Nat. Protoc 2009, 4, 1128-1144. [PubMed: 19617885]

(29). Farrar MJ; Bernstein IM; Schlafer DH; Cleland TA; Fetcho JR; Schaffer CB Chronic in Vivo Imaging in the Mouse Spinal Cord Using an Implanted Chamber. Nat. Methods 2012, 9, 297302. [PubMed: 22266542]

(30). Stiers P-J; van Gastel N; Moermans K; Stockmans I; Carmeliet G An Ectopic Imaging Window for Intravital Imaging of Engineered Bone Tissue. JBMR Plus 2018, 2, 92-102. [PubMed: 30283894]

(31). Meijer EFJ; Jeong H-S; Pereira ER; Ruggieri TA; Blatter C; Vakoc BJ; Padera TP Murine Chronic Lymph Node Window for Longitudinal Intravital Lymph Node Imaging. Nat. Protoc 2017, 12, 1513-1520. [PubMed: 28683064]

(32). Ritsma L; Steller EJA; Beerling E; Loomans CJM; Zomer A; Gerlach C; Vrisekoop N; Seinstra D; van Gurp L; Schäfer R; van Rheenen J; et al. Intravital Microscopy Through an Abdominal Imaging Window Reveals a Pre-Micrometastasis Stage During Liver Metastasis. Sci. Transl. Med 2012, 4, 158ra145-158ra145.

(33). Bochner F; Fellus-Alyagor L; Kalchenko V; Shinar S; Neeman M A Novel Intravital Imaging Window for Longitudinal Microscopy of the Mouse Ovary. Sci. Rep 2015, 5, 12446. [PubMed: 26207832]

(34). Stewart AJ; Blindauer CA; Berezenko S; Sleep D; Sadler PJ Interdomain Zinc Site on Human Albumin. Proc. Natl. Acad. Sci. U. S. A 2003, 100, 3701-3706. [PubMed: 12598656]

(35). Livieri M; Mancin F; Saielli G; Chin J; Tonellato U Mimicking Enzymes: Cooperation between Organic Functional Groups and Metal Ions in the Cleavage of Phosphate Diesters. Chem. - Eur. J 2007, 13, 2246-2256. [PubMed: 17163547]

(36). Major JL; Boiteau RM; Meade TJ Mechanisms of ZnII-Activated Magnetic Resonance Imaging Agents. Inorg. Chem 2008, 47, 10788-10795. [PubMed: 18928280]

(37). Gonzalez G; Powell DH; Tissieres V; Merbach AE Water-Exchange, Electronic Relaxation, and Rotational Dynamics of the MRI Contrast Agent [Gd(DTPA-BMA)(H2O)] in Aqueous Solution : A Variable Pressure, Temperature, and Magnetic Field 17 O NMR Study. J. Phys. Chem 1994, 98, 53-59.

(38). Powell DH; Dhubhghaill OMN; Pubanz D; Helm L; Lebedev YS; Schlaepfer W; Merbach AE Structural and Dynamic Parameters Obtained from 17O NMR, EPR, and NMRD Studies of Monomeric and Dimeric Gd3+ Complexes of Interest in Magnetic Resonance Imaging: An Integrated and Theoretically Self-Consistent Approach1. J. Am. Chem. Soc 1996, 118, 9333 9346.

(39). Martins AF; Morfin J-F; Geraldes CFGC; Tóth É Gd3+ Complexes Conjugated to Pittsburgh Compound B: Potential MRI Markers of $\beta$-Amyloid Plaques. JBIC, J. Biol. Inorg. Chem 2014, 19, 281-295. [PubMed: 24297602]

(40). Martins AF; Oliveira AC; Morfin J-F; Laurents DV; Tóth É; Geraldes CFGC Associating a Negatively Charged GdDOTA-Derivative to the Pittsburgh Compound B for Targeting A $\beta$ Amyloid Aggregates. JBIC, J. Biol. Inorg. Chem 2016, 21, 83-99. [PubMed: 26613605]

(41). Caravan P; Farrar CT; Frullano L; Uppal R Influence of Molecular Parameters and Increasing Magnetic Field Strength on Relaxivity of Gadolinium- and Manganese-Based T1 Contrast Agents. Contrast Media Mol. Imaging 2009, 4, 89-100. [PubMed: 19177472]

(42). Sherry AD; Wu Y The Importance of Water Exchange Rates in the Design of Responsive Agents for MRI. Curr. Opin. Chem. Biol 2013, 17, 167-174. [PubMed: 23333571]

(43). Stefan Y; Meda P; Neufeld M; Orci L Stimulation of Insulin Secretion Reveals Heterogeneity of Pancreatic B Cells in Vivo. J. Clin. Invest 1987, 80, 175-183. [PubMed: 3110211] 
(44). Zhu S; Larkin D; Lu S; Inouye C; Haataja L; Anjum A; Kennedy R; Castle D; Arvan P Monitoring C-Peptide Storage and Secretion in Islet $\beta$-Cells In Vitro and In Vivo. Diabetes 2016, 65, 699-709. [PubMed: 26647386]

(45). Martins EO; Drakenberg T Cadmium(II), Zinc(II), and Copper(II) Ions Binding to Bovine Serum Albumin. A 113Cd NMR Study. Inorg. Chim. Acta 1982, 67, 71-74.

(46). Sadler PJ; Viles JH $1 \mathrm{H}$ and 113Cd NMR Investigations of Cd2+ and Zn2+ Binding Sites on Serum Albumin: Competition with Ca2+, Ni2+, Cu2+, and Zn2+. Inorg. Chem 1996, 35, 44904496. [PubMed: 11666670]

(47). Lu J; Stewart AJ; Sadler PJ; Pinheiro TJT; Blindauer CA Albumin as a Zinc Carrier: Properties of Its High-Affinity Zinc-Binding Site. Biochem. Soc. Trans 2008, 36, 1317-1321. [PubMed: 19021548]

(48). Barg S; Eliasson L; Renstrom E; Rorsman P A Subset of 50 Secretory Granules in Close Contact With L-Type Ca2+ Channels Accounts for First-Phase Insulin Secretion in Mouse $\beta$-Cells. Diabetes 2002, 51, S74-S82. [PubMed: 11815462]

(49). Xue M; Giagtzoglou N; Bellen HJ Dueling Ca2+ Sensors in Neurotransmitter Release. Cell 2011, 147, 491-493. [PubMed: 22036557] 
(A)

(B)

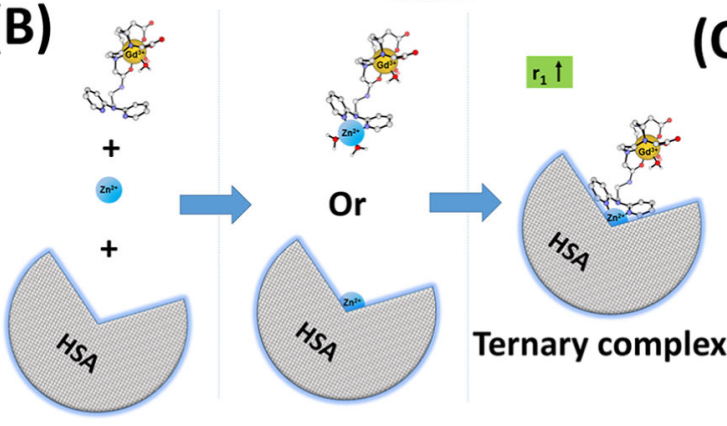

high Zn(II) affinity

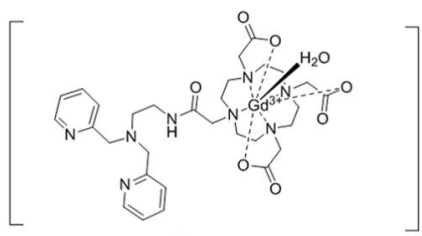

GdL1 lower Zn(II) affinity

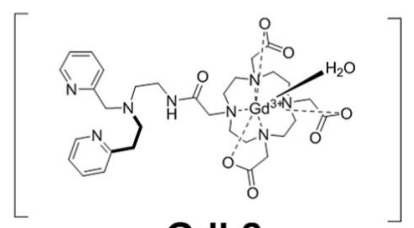

GdL2

C)

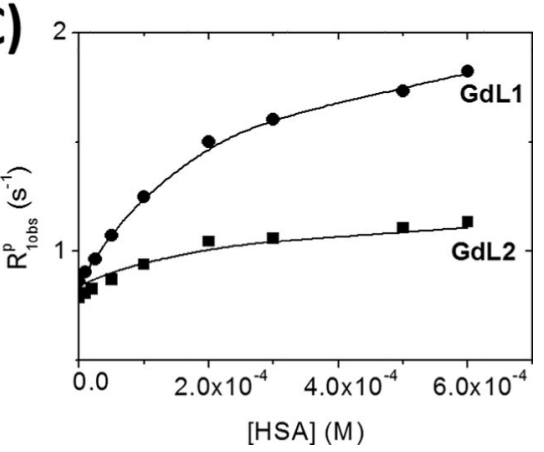

Figure 1.

(A) Chemical structures of GdDO3A-monoBPEN (GdL1) or monoPEPMA derivatives (GdL2). (B) Simplified illustration of the formation of a ternary complex, GdL-Zn-HSA. (C) Proton relaxation enhancement titrations of each complex (GdL1 and GdL2, $0.1 \mathrm{mM}$ ) as a function of increasing [HSA]. $\left[\mathrm{Zn}^{2+}\right]$ was held constant $(0.6 \mathrm{mM}$, equal to the highest concentration of HSA) in each titration. All measurements were performed at $0.5 \mathrm{~T}, 37^{\circ} \mathrm{C}$ in $100 \mathrm{mM}$ Tris buffer at $\mathrm{pH} 7$. 
A)

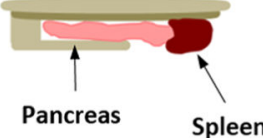

B)
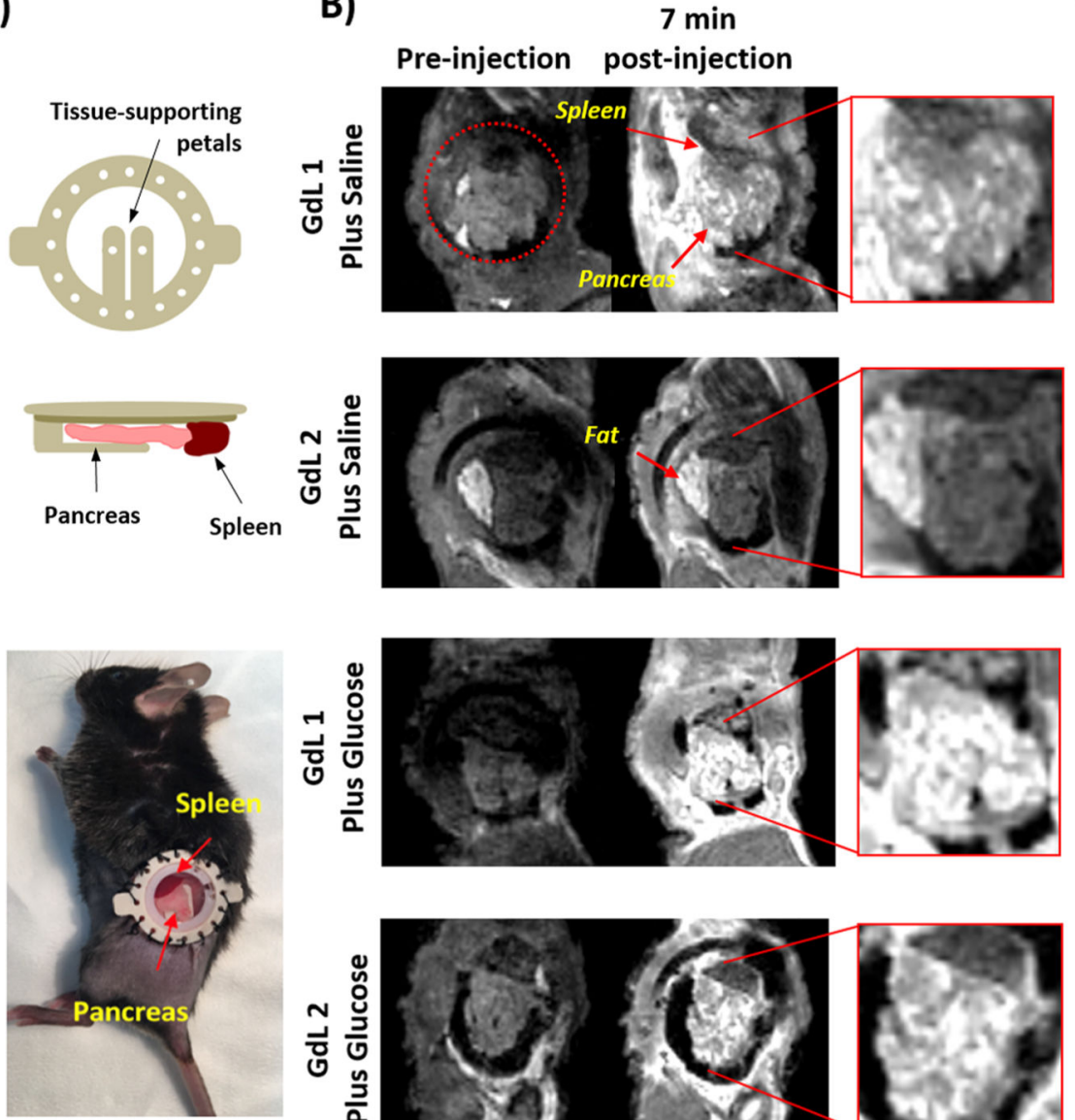

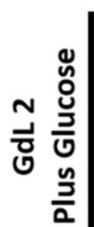

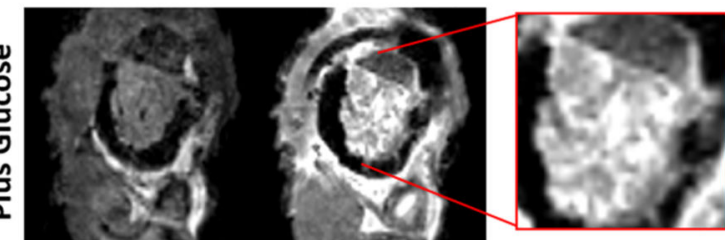
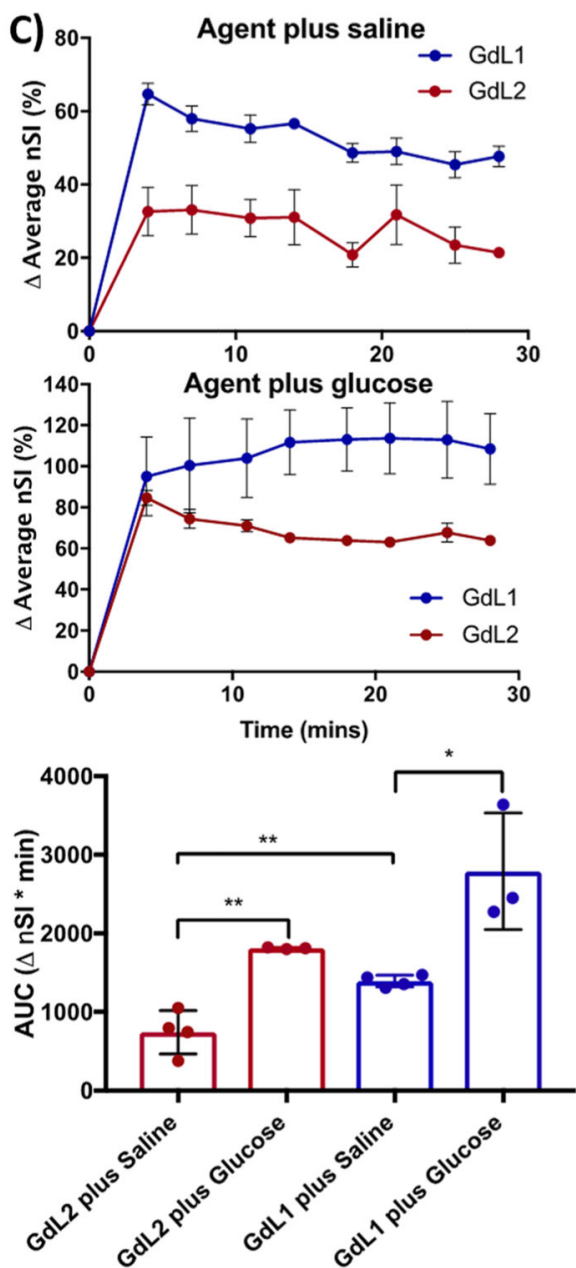

Figure 2.

Localized hotspots are observed in the pancreas tail by MRI after stimulation by glucose using an implanted imaging window to localize the mouse pancreas. (A) MR-compatible implantable window allowing the tail of the pancreas and spleen to be visible and held in place by the glass window coverslip. (B) 3D $T_{1}$-weighted MRI of mouse pancreas pre- and postdelivery of $0.07 \mathrm{mmol} / \mathrm{kg} \mathrm{GdL} 1$ or GdL2 plus saline or glucose. (C) Average MR signal intensity of the pancreas (compared to pre-injection baseline scans) after injection of either GdL1 or GdL2 agent plus saline (top panel). The middle panel shows the average signal of the pancreas (compared to preinjection baseline scans) after injection of either GdL1 or GdL2 agent plus glucose. The bottom panel shows the average area under the entire curve from 0 to $28 \mathrm{~min}$ for saline-treated mice $(N=4)$ and for glucose-treated animals $(N=3)$. Bars represent the standard error of the mean; ${ }^{*} p$-value $<0.05,{ }^{* *} p$-value $<0.01$. 
A)

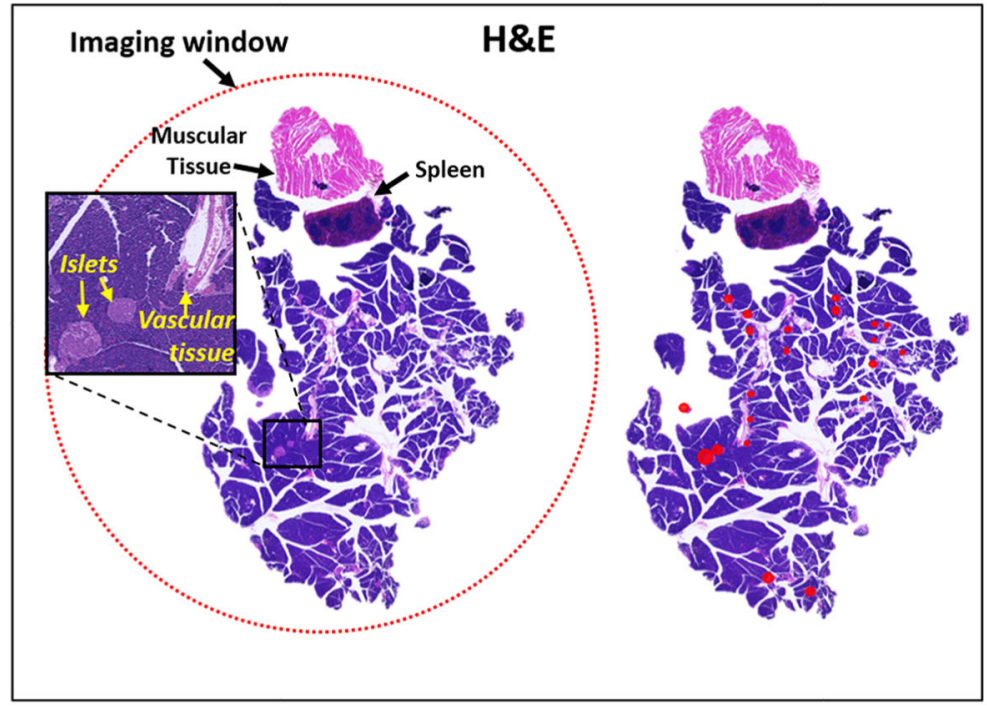

B)

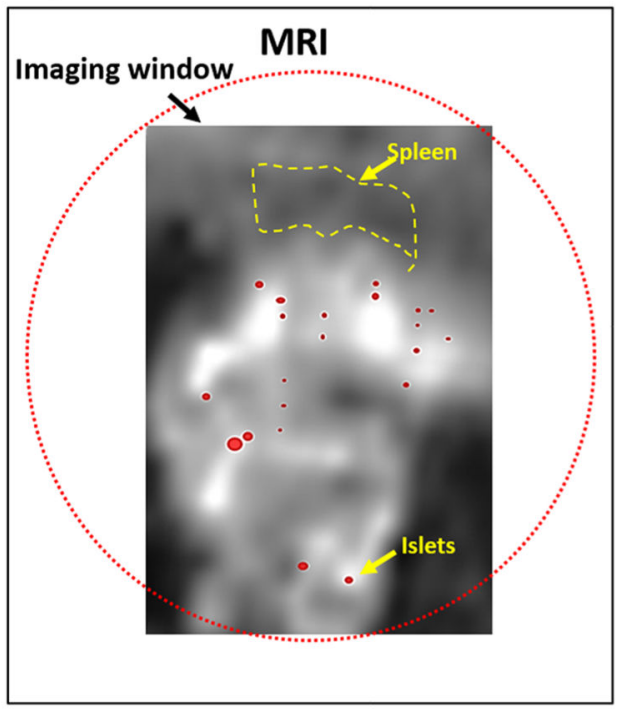

Figure 3.

Pancreatic islets in the tail of the pancreas colocalizes with MRI-observed glucosestimulated insulin secretion (GSIS) hotspots. (A) Mouse pancreas tail held by imaging window was resected and sectioned for histological staining. Islets were identified throughout the tissue (left) and labeled for visual identification (right). (B) MR image of a slice of tissue that closely matches the histological slice was collect 7 min after injection GdL2 and glucose. Labeled islets (red circles) from H\&E are shown as an overlay. 


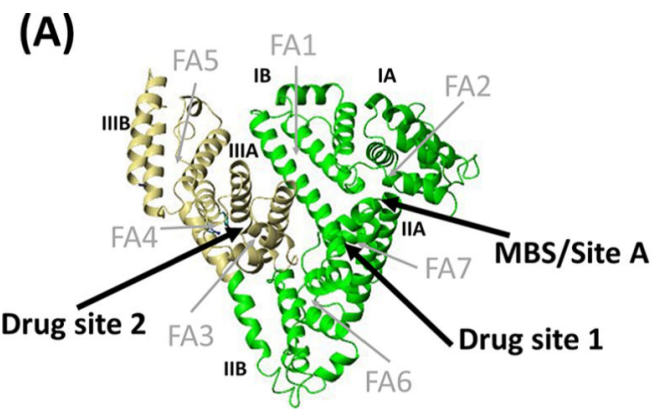

(B)

(C)
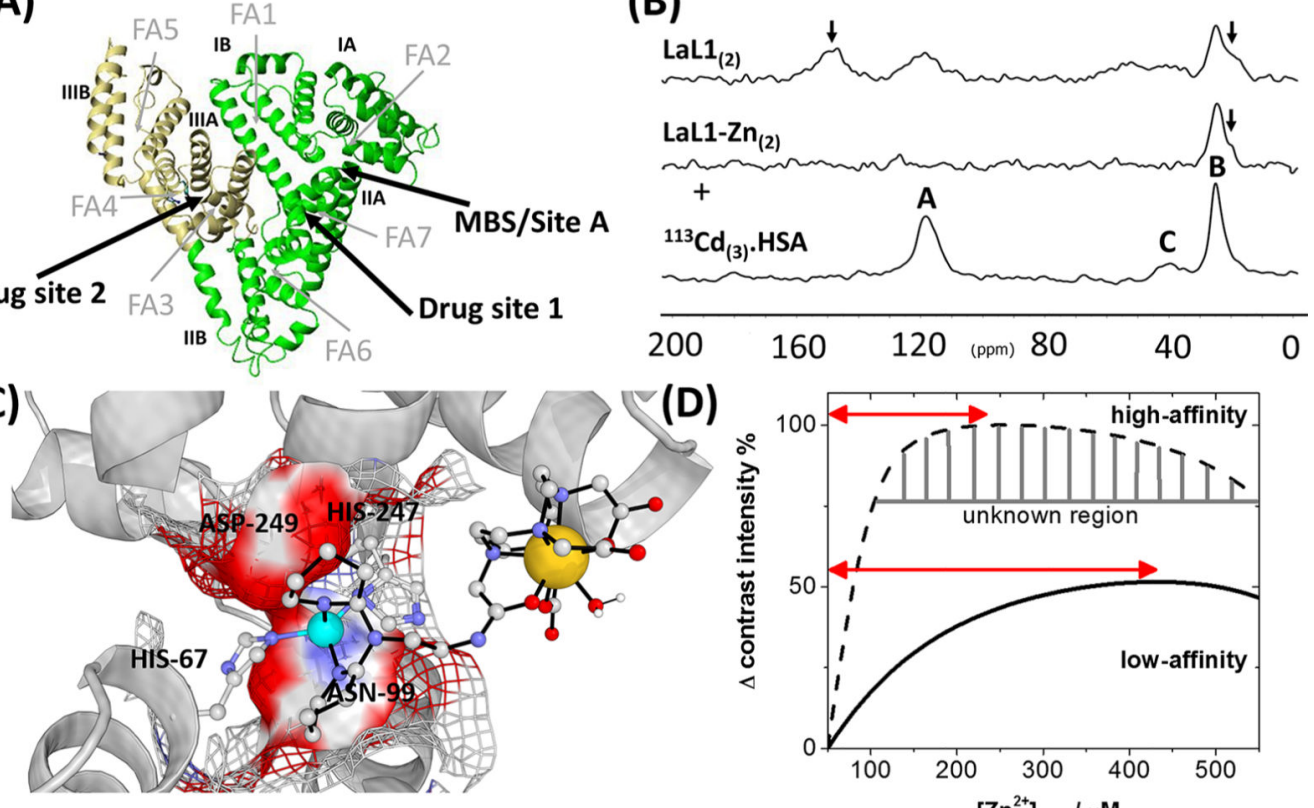

Figure 4.

Formation of the ternary complexes occurs mainly at the HSA MBS/site A, and the mathematical model predicts the expected contrast intensity signal. (A) Domain structure of albumin (PDB ID code 1AO6): domains I and II are colored green (residues 1-373), domain III is in yellow (residues 380-571), and long chain fatty acid sites (FA), Sudlow's drug binding sites, and zinc binding site A (MBS/site A) are also shown. (B) The $59.92 \mathrm{MHz}$ ${ }^{113} \mathrm{Cd}$ NMR spectra of HSA $(2 \mathrm{mM})$ in the presence of 3 mol equiv of ${ }^{113} \mathrm{CdCl}_{2}$, at $\mathrm{pD}=7.4$ (bottom) and after the addition of 2 mol equiv of LaL1-Zn (middle) and LaL1 alone (top) in Tris buffer. The black arrows point to two new ${ }^{113} \mathrm{Cd}$ sites that appear after addition of the agent. (C) HSA MBS/site A bound to Zn-GdL1: minimized MM+ structure involving zinc coordination with His-67, His247, and the two pyridines on the sensor. The surface charge is colored in red and blue for negative and positive charges, respectively. Zinc is colored light blue and gadolinium is gold. (D) Calculated plots of $\Delta$ contrast intensity (\%) vs total $\mathrm{Zn}(\mathrm{II})$ levels, starting at $50 \mu \mathrm{M}$ basal $\mathrm{Zn}^{2+}$ levels in the extracellular space around $\beta$-cells. The model assumes total extracellular $[\mathrm{GdL}]=100 \mu \mathrm{M}$ and total $[\mathrm{HSA}]=600 \mu \mathrm{M}$. The red arrows reflect the range of $\mathrm{Zn}^{2+}$ concentrations where the image contrast would remain sensitive to changes in $\left[\mathrm{Zn}^{2+}\right]$. 
Martins et al.

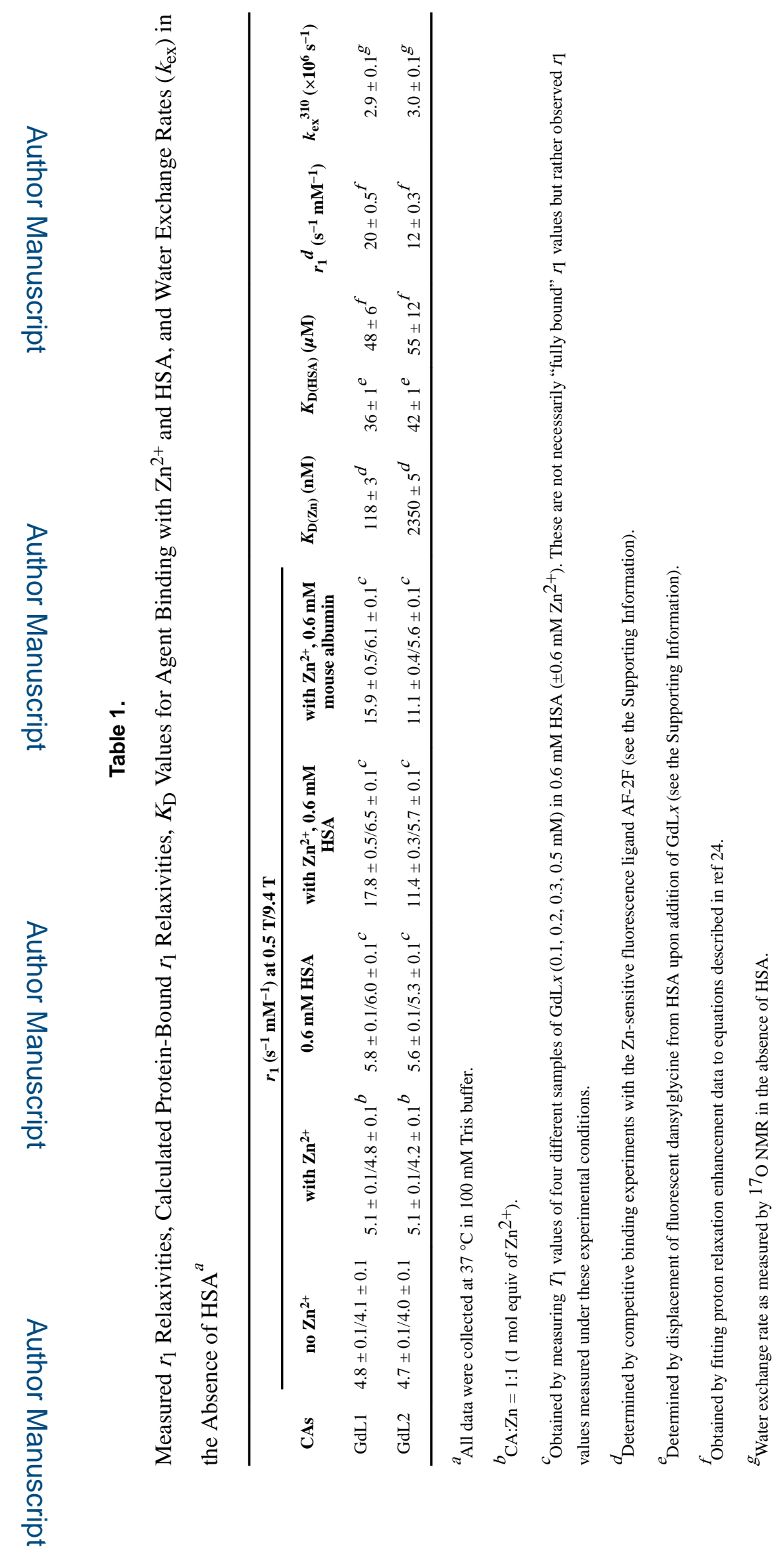

J Am Chem Soc. Author manuscript; available in PMC 2019 December 19. 